\title{
An Adaptive Modulation Scaling Scheme for Quality of Services Ensurance in Wireless Sensor Netwoks
}

\author{
${ }^{1}$ Yong Yuan, ${ }^{1}$ Zongkai Yang and ${ }^{2}$ Jianhua He \\ ${ }^{1}$ Department of Electronics and Information, Huazhong University of Science and Technology \\ Wuhan, China \\ ${ }^{2}$ Department of Electrical and Electronic Engineering, University of Bristol \\ Bristol, United Kindom
}

\begin{abstract}
Energy consumption has been a key concern of data gathering in wireless sensor networks. Previous research works show that modulation scaling is an efficient technique to reduce energy consumption. However, such technique will also impact on both packet delivery latency and packet loss, therefore, may result in adverse effects on the qualities of applications. In this study, the problem of modulation scaling and energy optimization is discussed. A mathematical model is proposed to analyze the impact of modulation scaling on the overall energy consumption, end-to-end mean delivery latency and packet loss. A centralized optimal management mechanism is developed based on the model, which adaptively adjusts the modulation levels to minimize energy consumption while ensuring the QoS for data gathering. Experimental results show that the management mechanism saves significant energy in all the investigated scenarios. Some valuable results are also observed in the experiments.
\end{abstract}

Key words: Wireless Sensor Network, Adaptive Modulation Scaling, Energy Aware Protocol Design

\section{INTRODUCTION}

Data gathering in the applications of wireless sensor network (WSN), especially in the military applications, normally requires a timely and reliable fashion. In order to maximize the lifetime of WSNs, it should also be carried out in an energy awareness manner. A lot of research efforts have been spent on minimizing latency and energy consumption in WSNs [1-4]. Energy awareness can be incorporated into the design of individual sensor, wireless communication between a pair of sensors and the operation of whole network [4]. Several techniques have been used in the level of individual sensor to optimize energy consumption, such as DVS and power management. Techniques such as topology management and reducing overhead are used in saving energy consumption in the level of whole network. Since wireless communication consumes a major portion of the overall network energy, schemes taking into account energy consumption of wireless communication will yield higher energy efficiency.

Modulation scaling is an efficient approach to adapting dynamic traffic loads by adjusting the modulation levels in the level of wireless communication. Recently this technique is utilized to reduce energy consumption in communications over wireless link [1, 2]. It is shown that the technique can be used to efficiently save energy with the introduction of some delivery latency. An optimization model is proposed to minimize the total energy consumption by selecting optimally modulation level of each node in a data aggregation tree [3]. The model traded off the end-to-end latency of the transmission in data aggregation tree and the energy consumption of each link in the tree. The limitation in the model is that only the latency introduced by transmission is considered. Latency introduced by queuing in each node is not considered. Due to the limited bandwidth and storage space, queuing latency will also be a serious problem and will result in packet dropping especially under heavy traffic load, such as in the video surveillance application [5]. On the other hand, $\mathrm{Yu}$ [3] only considered the tradeoff between energy consumption and end-to-end delivery latency, while end-to-end packet losses are also important for applications.

Limitations of the existing works motive us to design a more efficient modulation scaling control scheme with application qualities in mind. Energy consumption will be minimized based on modulation scaling, without violation requirements of end-to-end mean delivery latency and packet loss rate. As the channel quality and network traffic may vary significantly, the control scheme is also expected to be adaptive. In this study, the above problem is studied by abstracting the underlying structure of the network as a data aggregation tree. Based on classical queuing theory, an analytical model will be built in terms of modulation level of the nodes in the delivery path, overall latency and packet loss. The impacts of different settings of buffer size and packet arrival rates will also be considered in the analytical model. Then, optimal modulation levels for each node will be found to minimize energy consumption of the 
whole network. Constraints are imposed on the end-to-end latency and packet loss rate expected from applications. Then a centralized management mechanism can be developed based on the optimization model, which can adaptively adjust the modulation level of each node responding to the dynamic network conditions.

\section{MATERIALS AND METHODS}

Problem Statement: The central problem to be addressed in this work is how to determine the optimal modulation level for each node in WSN such that the overall energy consumption is minimized, without violating the application requirements on the mean end-to-end latency and packet loss ratio. The following assumptions are made to solve the problem without loss of generality.

1. Modulation levels can be adjusted for the wireless module of the node;

2. The buffer of each node can hold up to N packets;

3. The underlying topology structure of the network is abstracted as a data aggregation tree [6]. The time and energy costs for generating raw data at source nodes or aggregating data at internal nodes are considered to be negligible;

4. The resource and computational power of sink are unlimited and sink knows the structure of data aggregation tree and the packet arrival rates of all source nodes;

5. Each link in the data aggregation tree is assumed to be reliable;

6. Packet arrivals of source nodes follow Possion process. Packets size follows a negative exponential distribution, with mean of s;

7. For simplicity, the inference among nodes is assumed to be solved by spatial, frequency or code diversity [7] and no retransmission will be considered if packet is lost.

The data aggregation tree is denoted by $\mathrm{T}=\langle\mathrm{V}, \mathrm{E}\rangle$, where node set $\mathrm{V}$ denotes the set of all nodes in WSN. V is divided into source nodes set $V_{s}$, internal nodes set $V_{r}$ and sink, i.e. $\mathrm{V}=\mathrm{V}_{\mathrm{s}} \mathrm{UV} \mathrm{V}_{\mathrm{r}} \mathrm{U}\{$ sink $\}=\left\{\left\{\mathrm{S}_{1}, \mathrm{~S}_{2}, \ldots, \mathrm{S}_{\mathrm{m}}\right\},\left\{\mathrm{r}_{1}\right.\right.$, $\left.r_{2}, \ldots, r_{n}\right\}$, sink $\}$. For each source node $j$, we denote $L_{j}$ as the delivery path from source node $\mathrm{j}$ to sink in $\mathrm{T}$. We denote the packet arrival rate, packet service rate and packet output rate of node $\quad i \in V_{s} \cup V_{r} \quad$ as $\mathrm{u}_{\mathrm{j}}, \lambda_{\mathrm{j}}$ and $\mathrm{O}_{\mathrm{j}}$, respectively. Under the above assumptions, each node can be viewed as an M/M/1 queuing system with limited capacity from the viewpoint of queuing theory. So the packet arrival rate of the internal node is equal to the sum of packet output rates of its children (The impact of data aggregation is considered in the packet service rate). Then for the node $i \in V_{s} \cup V_{r}$ we denote the probability of queue being empty, queue being full and the mean time cost spent on queuing and transmitting a packet as $\mathrm{P}_{0}\left(\mathrm{u}_{\mathrm{j}}\right.$, $\left.\lambda_{\mathrm{j}}\right), \mathrm{P}_{\mathrm{N}}\left(\mathrm{u}_{\mathrm{j}}, \lambda_{\mathrm{j}}\right)$ and $\mathrm{W}_{\mathrm{i}}\left(\mathrm{u}_{\mathrm{j}}, \lambda_{\mathrm{j}}\right)$. They depend on $\mathrm{N}, \mathrm{u}_{\mathrm{i}}, \lambda_{\mathrm{i}}$. As presented later, solutions for $\mathrm{M} / \mathrm{M} / 1$ queuing system can be used directly to get the results on $\mathrm{P}_{0}, \mathrm{P}_{\mathrm{N}}$ and $\mathrm{W}_{\mathrm{i}}$ [8]. The node will output packets except that there are no packets in queue, so $\mathrm{O}_{\mathrm{i}}$ is equal to $\left(1-\mathrm{P}_{0}\right) \mathrm{u}_{\mathrm{i}}$. If the queue of the node is full, the node will discard the incoming packets. So the packet loss rate for node $\mathrm{i}$, denoted as $\mathrm{P}_{\mathrm{li}}$, is equal to $\mathrm{P}_{\mathrm{N}}$. On the other hand, for the node $i \in V_{s} \cup V_{r}$, its mean energy cost for transmitting a packet, denoted as $\mathrm{E}$, will be dependent on its modulation level, which is determined by $u_{i}$. Thus $E$ is a function of $u_{i}$, details of which will be provided in the next subsection.

With the above definitions, the end-to-end mean delivery latency and packet loss rate of the data from source node $\mathrm{j}$ are given by,

$$
W_{A j}=\sum_{k: V_{k} \in L j} W_{k} \quad P_{l A j}=1-\prod_{k: V_{k} \in L j}\left(1-P_{l k}\right)
$$

So the mean overall latency and packet loss ratio are

$$
W_{A}=\frac{\sum_{j \in V_{s}} \lambda_{j} W_{A j}}{\sum_{j \in V_{s}} \lambda_{j}} \quad P_{L A}=\frac{\sum_{j \in V_{s}} \lambda_{j} P_{L A j}}{\sum_{j \in V_{s}} \lambda_{j}}
$$

Then the problem can be modeled as an optimization problem, to find the optimal set of packet service rates $\left\{u_{j}\right\}$ so as to minimize energy consumption. It is formulated as follows.

Objective: $\min \sum_{j \in V s \cup V r} E\left(u_{j}\right)$

Constraints:
1. The mean overall latency should be less than $\Gamma, \mathrm{W}_{\mathrm{A}} \leq \Gamma$.

2. The mean overall packet loss ratio should be less than $\mathrm{P}_{\text {loss }}, \mathrm{P}_{\mathrm{LA}} \leq \mathrm{P}_{\text {loss }}$.

Optimization Model and Corresponding Management Mechanism: In this subsection, we will analyze the relationship among the amount of latency, packet loss introduced by each node, its packet service rate and energy consumption. An optimization model to solve the optimization problem will be developed based on these relationships. A centralized management mechanism will also be proposed to adaptively adjust the modulation level of each node so as to save energy without violating the constraints on latency and packet loss in dynamic environments.

As we analyzed, each node can be treated as an M/M/1 queuing system with limited capacity. Thus the relationship between latency, packet loss introduced by each node and its packet service 
rate can be analyzed by classic queuing theory [8]. The existing solutions will be directly used in the study.

Relationship Between Packet Service Rate and Energy Consumption E(u): It's observed by Schurgers et al. [9] that energy consumed in transmitting one packet is $\mathrm{E}=\left[\mathrm{C} \cdot\left(2^{\mathrm{b}}-1\right)+\mathrm{F}\right] \cdot \mathrm{m} \cdot \mathrm{R}$, where $\mathrm{C}, \mathrm{F}$ are constants determined by the energy model and $\mathrm{b}, \mathrm{R}, \mathrm{m}$ are respectively modulation level, symbol rate and mean service time for one packet. The mean service time of source nodes, i.e. $1 / \mathrm{u}$, for one packet equals to $\mathrm{s} / \mathrm{bR}$. The mean service time of internal nodes depends on not only the transmission speed but also the amount of output data for a packet after aggregation. The amount of output data is dependent on the correlations of data before aggregation. We adopt the aggregation model [3] and use the aggregation factor to describe the correlations among data. Assuming the degree of correlations among data before aggregation is indicated by aggregation factor $\mathrm{k}$, the amount of output data for each packet before aggregation is $\mathrm{s} /(\mathrm{dk}-\mathrm{k}+1)$ and the mean service time, i.e. $1 / \mathrm{u}$, is $\mathrm{s} /((\mathrm{dk}-\mathrm{k}+1) \mathrm{bR})$. $\mathrm{d}$ is number of packets to aggregate. So the relationship between the mean energy cost and the packet service rate is

$$
E(y)=\left[C \bullet\left(2^{\frac{s u}{(d k-k+1) R}}-1\right)+F\right] \bullet \frac{1}{u} \bullet R
$$

For any source node, we have $\mathrm{d}=1, \mathrm{k}=0$, as internal node $\mathrm{d}$ is the number of its children, $\mathrm{k}$ is the aggregator factor. Figure 1 plots the energy function with $\mathrm{d}=2, \mathrm{k}=0.5$, $\mathrm{F}=1 \mathrm{e}-6, \mathrm{C}=6.67 \mathrm{e}-9$, and $\mathrm{u}$ is chosen from $[5,140]$.

From Fig.1, it's observed that the function E(u) is concave upward. And the minimum of $\mathrm{E}(\mathrm{u})$ is gained at $\mathrm{u}=\mathrm{u}_{\min }$. If we choose $\mathrm{u}$ less than $\mathrm{u}_{\min }$, no energy savings will be gained but the service rate will be less. So u should be larger than $u_{\min }$. On the other hand, the modulation level is typically set to positive even integers, such as 4,8 , etc and less than 16 for the low-end wireless systems [4]. So u should be chosen from $\left\{u_{b}: b=2,4 \ldots 16\right.$, $\left.\mathrm{u}_{\mathrm{b}}=(\mathrm{dk}-\mathrm{k}+1) \mathrm{bR} / \mathrm{s}\right\}$.

Optimization Model: With the above results, the optimization model presented before can be rewritten as below. We can get the solution for the optimization model by classic optimization techniques. Based on the solution, the optimization model can be utilized further in the centralized control scheme.

\section{Definitions:}

The node set, $\mathrm{V}=\mathrm{V}_{\mathrm{s}} \mathrm{UV} \mathrm{V}_{\mathrm{r}} \mathrm{U}\{$ sink $\}=\left\{\left\{\mathrm{S}_{1}, \mathrm{~S}_{2}, \ldots, \mathrm{S}_{\mathrm{m}}\right\}, \quad\left\{\mathrm{r}_{1}\right.\right.$, $\left.\mathrm{r}_{2}, \ldots . ., \mathrm{r}_{\mathrm{n}}\right\}$, sink $\}$

The packet arrival rate for node $j$,

$$
\lambda_{j}=\left\{\begin{array}{l}
\sum_{k \in V_{s j}} O_{k}, j \in V r \\
\lambda_{j}, j \in V_{s}
\end{array}\right.
$$

The packet output rate for node $\mathrm{j}$,

$$
O_{j}=\left(1-P_{0}\left(u_{j}, \lambda_{j}\right)\right) u_{j}
$$

$P_{0}\left(u_{j}, \lambda_{j}\right)$ : The probability of queue empty for node $j$.

$\mathrm{P}_{\mathrm{lj}}\left(\mathrm{u}_{\mathrm{j}}, \lambda_{\mathrm{j}}\right)=\mathrm{P}_{\mathrm{N}}\left(\mathrm{u}_{\mathrm{j}}, \lambda_{\mathrm{j}}\right)$ : The probability of queue full for node j. i.e. the probability of packet loss.

$\mathrm{L}_{\mathrm{j}}\left(\mathrm{u}_{\mathrm{j}}, \lambda_{\mathrm{j}}\right)$ :The average number of packets(including the packets in waiting and service) for node $\mathrm{j}$.

$\mathrm{W}_{\mathrm{j}}\left(\mathrm{u}_{\mathrm{j}}, \lambda_{\mathrm{j}}\right)$ : The mean time cost for a packet to queue and transmit in node $\mathbf{j}$.

$E\left(u_{j}\right)$ : The mean energy cost for transmitting a packet in node $j$, whose packet service rate is $u_{j}$.

$\mathrm{W}_{\mathrm{Aj}}$ : The mean end-to-end latency of the data from source node $\mathrm{j}$.

$\mathrm{P}_{\mathrm{LAj}}$ : The mean end-to-end packet loss ratio of the data from source node $\mathrm{j}$.

$\mathrm{W}_{\mathrm{A}}$ : The mean overall latency.

$\mathrm{P}_{\mathrm{LA}}$ : The mean overall packet loss ratio.

Objective:

$$
\begin{aligned}
& \min \sum_{j \in V s \cup V r} E\left(u_{j}\right) \\
& =\min \sum_{j \in V s \cup V r}\left[C \bullet\left(2^{\frac{s u_{j}}{(d k-k+1) R}}-1\right)+F\right] \bullet \frac{1}{u_{j}} \bullet R
\end{aligned}
$$

\section{Constraints:}

1. The mean overall latency should be less than $\Gamma, \mathrm{W}_{\mathrm{A}} \leq \Gamma$.

2. The mean overall packet loss ratio should be less than $\mathrm{P}_{\text {loss }}, \mathrm{P}_{\mathrm{LA}} \leq \mathrm{P}_{\text {loss }}$.

3. $\mathrm{u}\left\{\mathrm{u}_{\mathrm{b}}: \mathrm{b}=2,4, \ldots, 16, \mathrm{u}_{\mathrm{b}}=(\mathrm{dk}-\mathrm{k}+1) \mathrm{bR} / \mathrm{s}\right.$ and $\mathrm{u}_{\mathrm{b}}$ $\left.\geq u_{\min }\right\}$.

Expected solution: Find the optimal set of $\left\{\mathrm{u}_{\mathrm{j}}^{*}\right\}$.

Centralized Management Mechanism: A centralized management mechanism is then developed based on the optimization model. It is designed to adaptively adjust the modulation level of each node so as to save energy without violating the constraints on latency and packet loss in dynamic environments. There are three key procedures in the mechanism. First, after constructing the data aggregation tree, the information about the structure of the tree is sent to the sink. Second, the packet arrival rate of each source node is measured periodically and sent to the sink if there is a large change. Third, the sink finds the optimal modulation level for each node in the path based on the optimization model and sends information back to the nodes, which will adjust modulation level correspondingly. If the network conditions change a lot, then the sink will calculate again the optimal modulation levels. 


\section{RESULTS AND DISCUSSION}

In order to evaluate the performance of the optimization management scheme, we conduct experiments in MATLAB. Without loss of generality, we assume the data aggregation tree is a complete binary tree with 7 nodes in the experiments. Other related parameters are set as follow, $\mathrm{F}=1 \mathrm{e}-6, \mathrm{C}=6.67 \mathrm{e}-9, \mathrm{R}=10 \mathrm{kbps}, \mathrm{s}=1 \mathrm{kbit}$, $\mathrm{N}=20, \lambda_{1}=40, \lambda_{2}=50, \lambda_{3}=70$ and $\lambda_{4}=30$.

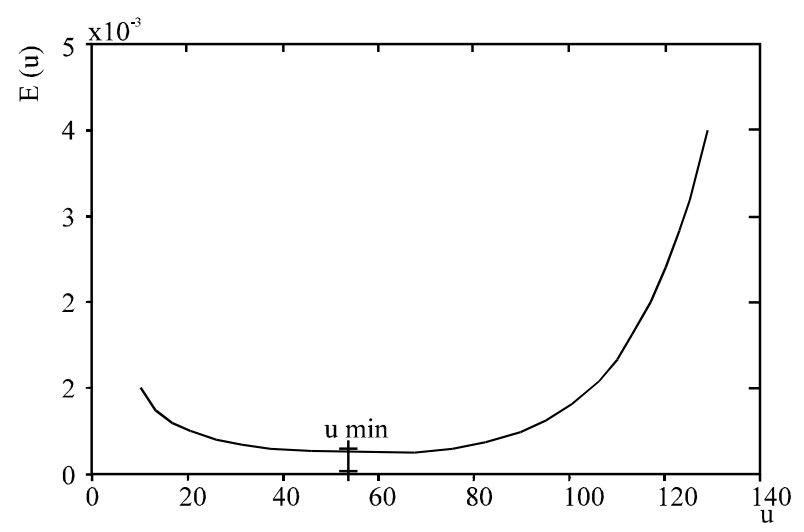

Fig. 1: Energy Function for Transmitting One Packet

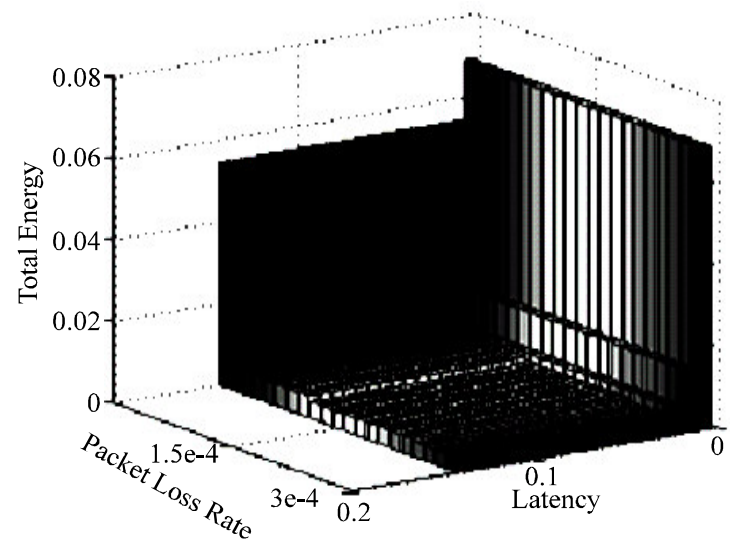

Fig. 2: Total Energy Versus QoS Requirements

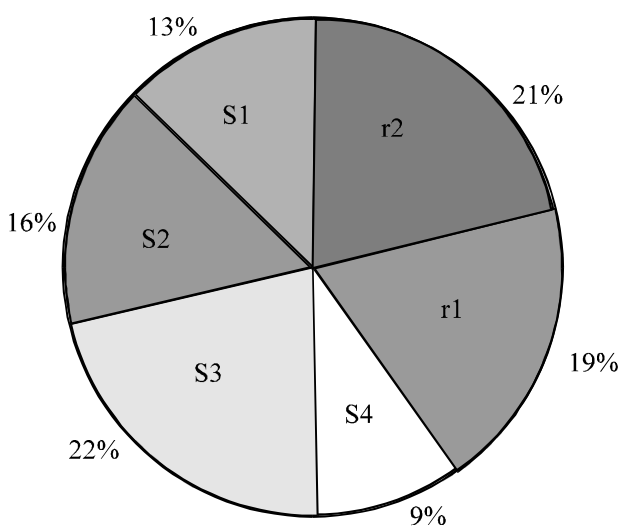

Fig. 3: Pie of Arrival Rate
In the experiments, we vary the latency constraint from $20 \mathrm{~ms}$ to $200 \mathrm{~ms}$ and the packet loss rate constraint from $6 \times 10^{-8}$ to $3 \times 10^{-4}$; and search the minimum energy consumption and optimal set of modulation levels by our model. Figure 2 plots the typical results on total energy consumption in these scenarios.

From the experimental results including those shown in Fig. 2, it's observed that, under different constraints on

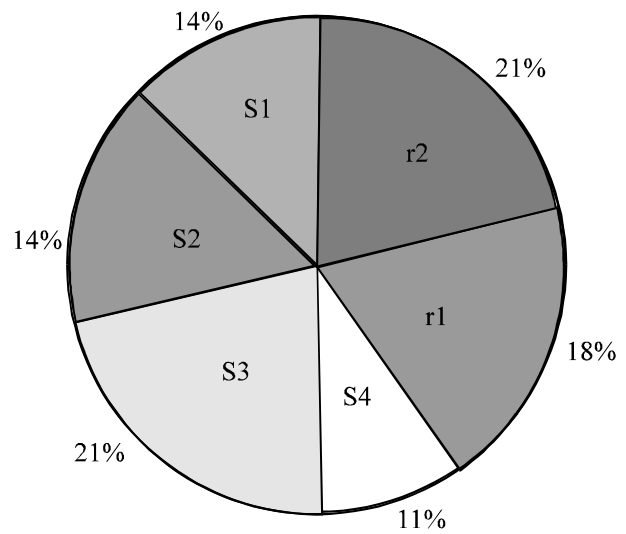

Fig. 4: Pie of Service Rate in Case 1

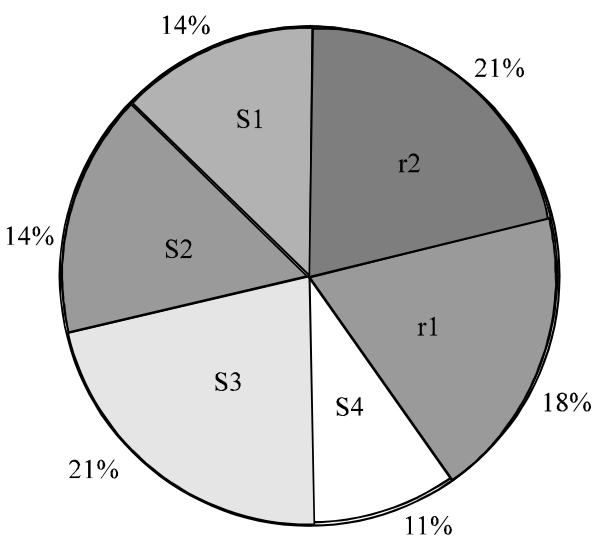

Fig. 5: Pie of Service Rate in Case 2

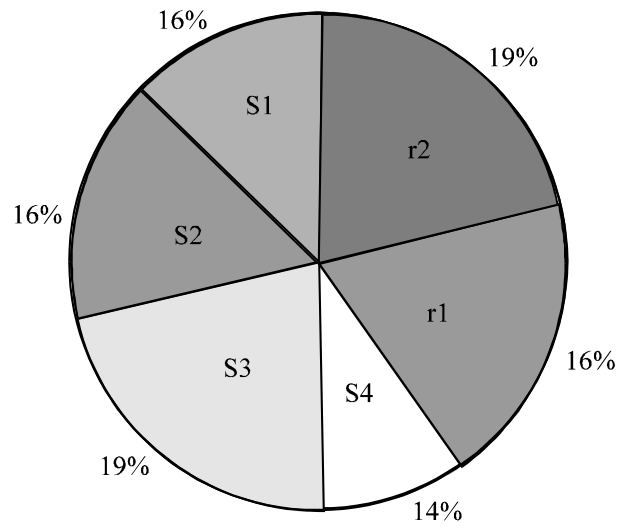

Fig. 6: Pie of Service Rate in Case 3 
latency and packet loss, the minimized energy consumption varies significantly. If the modulation levels are not to be adjusted dynamically, either more energy will be wasted or the requirements on end-to-end delivery quality will be violated. Furthermore, we observed that there exist critical ranges for constraints on latency and packet loss, which have significant impacts on the overall energy consumption. The range for constraint on latency in our experimental setting is $(0,80$ $\mathrm{ms}$ ) and the range for constraint on packet loss in our experimental setting is $(0,1.2 \mathrm{e}-4)$. As shown in Fig. 2, if the constraints are not in these ranges, the energy consumption can be saved so greatly by adjusting the modulation levels according to our model.

Another interesting finding from experiment results is that the optimal packet service rate of each node is proportional to the packet arrival rate in most scenarios. In order to describe this result, we plot the pie of packet arrival rates of the nodes in Fig. 3. The packet arrival rate of the internal node $\mathrm{j}$ is estimated as:

$\lambda_{j}=\frac{\sum_{l \in S_{j}} \lambda_{l}}{d k+k-1}$

Figure 4 to 6 plot the pie of the optimal packet service rates of the source nodes and internal nodes in three random scenarios of QoS requirements.

From Fig. 3 to 6, we can see the relative proportion of optimal packet service rates is similar to the relative proportion of packet arrival rates. So a simple way to trade off the energy consumption and the delivery quality is to set the packet service rate of each node proportional to its packet arrival rate. It will improve the performance of network in most scenarios.

\section{CONCLUSION}

In this study, we studied the problem of adaptive modulation scaling to minimize energy consumption without violating the end-to-end mean delivery latency and packet loss constraints of applications. We abstracted the underlying structure of the network as a data aggregation tree. Based on classical queuing theory, we analyzed the relationship among the modulation levels of the nodes in the delivery path, the overall latency and packet loss with different settings of buffer size and packet arrival rates. Then we developed an optimization model to find the optimal modulation level based on these results. A centralized management mechanism was proposed to adaptively adjust the modulation level.
Experimental results show that the model can save energy significantly in most investigated scenarios. Critical ranges of constraints for the latency and packet loss were observed which had large impact on energy consumption. We also found that the optimal packet service rate of each node was almost proportional to the packet arrival rate. Our future work will consider the problems of distributed control mechanism to adaptively adjust the modulation levels and the optimal packet scheduling in multiple sink scenarios.

\section{REFERENCES}

1. Prabhakar, B., E. Uysal-Biyikoglu and A.E. Gamal, 2001. Energy-efficient transmission over a wireless link via lazy packet scheduling. In Proceedings of IEEE INFOCOM, Anchorage, Alaska, pp: 386-394.

2. Gamal, A.E., C. Nair, B. Prabhakar, E. Uysal-Biyikoglu and S. Zahedi, 2002. Energy-efficient scheduling of packet transmissions over wireless networks. In Proceedings of IEEE INFOCOM, New York, USA, pp: 1773-1782.

3. Yu, Y., B. Krishnamachari and V.K. Prasanna, 2004. Tradeoffs for data gathering in wireless sensor networks. To appear in IEEE INFOCOM.

4. Raghunathan, V., C. Schurgers, S. Park and M. Srivastava, 2002. Energy-aware wireless microsensor networks. IEEE Signal Process. Mag., pp: 40-50.

5. Foresti, G.L. and L. Snidaro, 2002. A distributed sensor network for video surveillance of outdoor environments. In Proceedings of International Conference on Image Processing, (ICIP '02), New York, USA., 1: 22-25.

6. Krishnamachari, B., D. Estrin and S. Wicker, 2002. The impact of data aggregation in wireless sensor networks. In Proceedings of International Workshop on Distributed Event-Based Systems, (DEBS '02), Vienna, Austria, pp: 575-578.

7. Roy, S. and H.Y. Wang, 1999. Performance of CDMA slotted ALOHA multiple access with multiuser detection. In Proceedings of IEEE WCNC, 2: 839-843.

8. Takacs, L., 1962. Introduction to the Theory of Queues, Published by Oxford University Press, New York.

9. Schurgers, C., O. Aberborne and M. Srivastava, 2001. Modulation scaling for energy-aware communication systems. ISLPED, pp: 96-99. 